\title{
Information Content of Polarization Measurements
}

\author{
D G Ireland* \\ Department of Physics and Astronomy, \\ University of Glasgow, Glasgow G12 8QQ, United Kingdom
}

\begin{abstract}
Information entropy is applied to the state of knowledge of reaction amplitudes in pseudoscalar meson photoproduction, and a scheme is developed that quantifies the information content of a measured set of polarization observables. It is shown that this definition of information is a more practical measure of the quality of a set of measured observables than whether the combination is a mathematically complete set. It is also shown that when experimental uncertainty is introduced, complete sets of measurements do not necessarily remove ambiguities, and that experiments should strive to measure as many observables as practical in order to extract amplitudes.
\end{abstract}

PACS numbers: 13.60.Le,89.70.Cf

*Electronic address: d.ireland@physics.gla.ac.uk 


\section{INTRODUCTION}

In pseudoscalar meson photoproduction, the reaction is completely described by four amplitudes that are functions of hadronic mass $W$ and center of mass scattering angle $\theta_{C M}$ (or, equivalently $s$ and $t$ ). If one were able to extract these amplitudes (allowing of course for an overall phase) at $\left\{W, \cos \theta_{C M}\right\}$ or $\{s, t\}$ points, there is nothing else one could measure that would alter how one could interpret the physics of the reaction.

This observation is especially important in the study of the spectrum of baryon resonances. Despite several decades of investigation, it is still not clear whether certain states that are predicted by quark models exist or not. The signatures of any hitherto undiscovered states must be very subtle, to the extent that they are not readily apparent from cross section measurements alone. If one could unpick the reaction amplitudes from suitable observables, that would constitute the most comprehensive test for models. In the case of establishing s-channel resonances, extraction of the four amplitudes may not even be enough. Partial-wave analyses will be required, and these can lead to finite ambiguities that require additional information to resolve. In any event, a potential new physical effect would have to manifest itself clearly, or be declared unproven.

In order to extract the amplitudes, it is necessary to measure several polarization observables. In addition to the cross section, there are three single-spin observables [6]: $B$ (photon beam asymmetry), $R$ (recoil polarization) and $T$ (target polarization), which can be labelled as $\mathcal{S}$-type measurements. There are also four beam-recoil ( $\mathcal{B} \mathcal{R}$-type), four beam-target ( $\mathcal{B} \mathcal{T}$-type) and four recoil-target ( $\mathcal{R} \mathcal{T}$-type) observables. All these observables are bilinear combinations of the four reaction amplitudes, and are not independent. In principle, therefore, it is not necessary to measure all of them to be able to infer the amplitudes. As we have now entered an era in which single- and double-polarization measurements can be made, there exists a real opportunity for progress in understanding pseudoscalar meson photoproduction reactions, and for potential discovery of new states.

The problem of finding a minimum set of measurements that allows the unambiguous extraction of amplitudes was addressed by Barker, Donnachie and Storrow [1]). They found that, in addition to the single polarization set, five more double polarization observables were needed to remove all ambiguities in the quadrants for each relative phase 
angle. More recently, Chiang and Tabakin [2] carried out a detailed analysis of the algebra of observables using Fierz identities, and showed that the selection of just four suitably chosen double polarization observables was sufficient to remove the ambiguities. Such sets have been designated as "complete" sets.

The Fierz identity analysis led to a large number of identities among observables. Work by Artru et al. [4, 5] extended this by using positivity constraints to derive many inequalities. This means that the measurement of a subset of observables places limits on the possible values of the undetermined observables, so the inequalities provide useful guides to whether the values of experimental data are physical.

Labelling sets of observables as "complete", implies somehow that one has reached an ultimate state of knowledge. However, the reality is that all experimental measurements of observables carry with them a finite uncertainty, so the concept of completeness is not well defined. One might be tempted to regard this as an experimental failing, but in practice any experiment has to be performed within constraints of time and technological feasibility; the experiment with zero uncertainty can only be accomplished in an infinite time. The alternative is to embrace experimental uncertainty and include it in the interpretation of results.

The problem of uncertainty due to noise in communication channels led Shannon to develop the foundations of information theory [3]. In that seminal work, the concept of entropy was used as a means of quantifying an amount of information. One can also apply this to measurements. To introduce the idea with a concrete example, suppose one measured a quantity $X$ and obtained a measured value $x$ with an uncertainty $\delta_{x}$. The reporting of this measurement would usually be in the form $x \pm \delta_{x}$, but this is really shorthand for a Gaussian probability density function (PDF) $p(x)$. The entropy is then

$$
H=-\int p(x) \log p(x) d x,
$$

which for a Gaussian PDF is $H=\log \sqrt{2 \pi e}+\log \delta_{x}$. If a more accurate measurement were to be made, resulting in a reduced uncertainty $\delta_{x}^{\prime}$, the gain in information can be quantified as

$$
I=H-H^{\prime}=\log \left(\frac{\delta_{x}}{\delta_{x}^{\prime}}\right) .
$$

By extending this idea to the uncertainty in the reaction amplitudes, it is possible to quantify how much information is gained following the measurement of one or more ob- 
servables. This article represents a preliminary study of information entropy as applied to pseudoscalar meson photoproduction. Section II develops the idea encapsulated by Eq. (1) for the reaction amplitudes, and introduces a means of calculating it. In section III] examples of hypothetical measurements are given, which show how the magnitudes and relative phases of the amplitudes can be determined. In addition to this, section IV briefly considers how the information content of measured data can be used as a guide to estimating whether the measurement could in principle reduce uncertainty in derived physical quantities.

\section{MEASURING INFORMATION}

\section{A. Reduced Amplitudes}

A full analysis of reactions will involve measurements over all scattering angles and cover the mass range of interest. To develop the concept of information content, however, we restrict ourselves to considering one region (or "bin") in $\left\{W, \theta_{C M}\right\}$ space. The ideas can be straightforwardly extended to include many regions, since entropies are additive. The issue of whether different experiments (measuring different observables) have covered the same $\left\{W, \theta_{C M}\right\}$ space has been avoided.

The choice of basis for amplitudes is arbitrary; information content is derived from the measured observables, so it cannot depend on the choice. In this work, the transversity basis is used, where the spin of the target nucleon and recoiling baryon is projected onto the normal to the scattering plane, and the linear polarization of the photon is either normal or parallel to the scattering plane.

It is assumed that differential cross section measurements have been performed to a level of accuracy of, say, a few percent, so that further measurement would be unlikely significantly to improve knowledge of the amplitudes. The information gain to be studied here is solely due to an increased accuracy in the knowledge of the polarization observables. Since all these observables are asymmetries, no generality is lost if we rescale the amplitudes $b_{i} \rightarrow a_{i}$ such that

$$
a_{i}=\frac{b_{i}}{\sqrt{\left|b_{1}\right|^{2}+\left|b_{2}\right|^{2}+\left|b_{3}\right|^{2}+\left|b_{4}\right|^{2}}}
$$


so that the cross section provides an overall scale factor. Applying this rescaling, we have

$$
\left|a_{1}\right|^{2}+\left|a_{2}\right|^{2}+\left|a_{3}\right|^{2}+\left|a_{4}\right|^{2}=1
$$

Since these reduced amplitudes $a_{i}$ are complex, this represents the equation of a unit 7sphere, i.e. the eight numbers that are the real and imaginary parts are constrained to be on the surface of a unit hypersphere in 8 dimensions (a unit 8-ball).

The definitions of the observables in terms of the reduced amplitudes are given in appendix $\mathrm{A}$. One side-effect of choosing transversity amplitudes is that measurement of the $\mathcal{S}$-type observables leads to the extraction of the magnitudes, leaving just the relative phases to be determined. There is often a tacit assumption that it is easier to perform single-spin asymmetry measurements. For that reason many analyses [1, 2] start from a point where values of the $\mathcal{S}$-type observables have been determined.

\section{B. Entropy}

The entropy associated with the state of knowledge of the amplitudes is an multidimensional extension of Eq. (1):

$$
H=-\int p\left(\left\{x_{i}\right\}\right) \log p\left(\left\{x_{i}\right\}\right) d\left\{x_{i}\right\},
$$

where $\left\{x_{i}\right\}$ represents the values of the real and imaginary parts of the amplitudes. Before the measurement of any polarization observable, there is no knowledge of $\left\{x_{i}\right\}$, other than the constraint imposed by Eq. (2). To encode this as a PDF, we can spread the probability uniformly over the surface area of the unit 7-sphere to give

$$
p\left(\left\{x_{i}\right\}\right)=\frac{3}{\pi^{4}},
$$

which results in a pleasingly simple entropy of

$$
H_{7-\text { sphere }}=-\int \frac{3}{\pi^{4}} \log \left(\frac{3}{\pi^{4}}\right) d\left\{x_{i}\right\}=4 \log \pi-\log 3 .
$$

The act of measurement can be viewed as a compression of this "uniform" PDF into as small a region of $\left\{x_{i}\right\}$ space as possible. As a rough example, consider a set of measurements that results in a multi-dimensional Gaussian PDF in amplitude space. The entropy of an $n$-dimensional Gaussian is [3]

$$
H_{g}=\frac{n}{2} \log (2 \pi e)+\frac{1}{2} \log \left(\left|c_{i j}\right|\right)
$$


where $\left|c_{i j}\right|$ is the determinant of the covariance matrix. While the four complex amplitudes have eight numbers in total, representing real and imaginary parts, all observable quantities are invariant to the choice on an overall phase angle, so the effective number of numbers to extract is seven. In this case, a 7-dimensional Gaussian is used to estimate information gain. The projection of the Gaussian onto the 7-sphere will induce off-diagonal correlations in $c_{i j}$, but for simplicity we ignore any correlations and take the standard deviation in each of the $\left\{x_{i}\right\}$ to be the same ( $\sigma$, say). The resulting approximate expression is

$$
H_{\text {measured }}=\frac{7}{2} \log (2 \pi e)+7 \log \sigma .
$$

The gain in information is the difference between this and the initial uniform PDF over the 7-sphere:

$$
I=H_{7-\text { sphere }}-H_{\text {measured }}=4 \log \pi-\log 3-\frac{7}{2} \log (2 \pi e)-7 \log \sigma .
$$

A plot of this quantity as function the size of standard deviation is shown in Fig. (1). The choice of logarithm base is arbitrary, but for this work we select it to be 2 . This means that the unit of information is the "bit" (i.e. knowing whether a quantity is 1 or 0 ). This unit system is convenient for considering quantities related to polarization; determining whether an asymmetry is positive or negative is equivalent to a gain of one bit of information, whereas determining a phase angle quadrant is a gain of two bits.

From Fig. (1) it can be seen that if one wants to have a measured accuracy of the amplitudes to a value $\sigma=0.05$, the gain in information is roughly 21 bits (see dashed vertical line on graph). Attempting to achieve much better accuracy than this from experiments is not likely to be practical, so we should therefore regard the 21-bit information gain as a target figure to aim for, if we want to be able to say that we have extracted amplitudes. Furthermore, if two models differ by only a few percent in the values of their amplitudes, it is not reasonable to expect that comparison with data would ever lead to being able to differentiate them.

\section{Numerical calculation of entropies.}

While the calculation sketched out above is a useful rough guide, when an actual set of observables have been measured, Eq. (3) will need to be evaluated numerically. The 


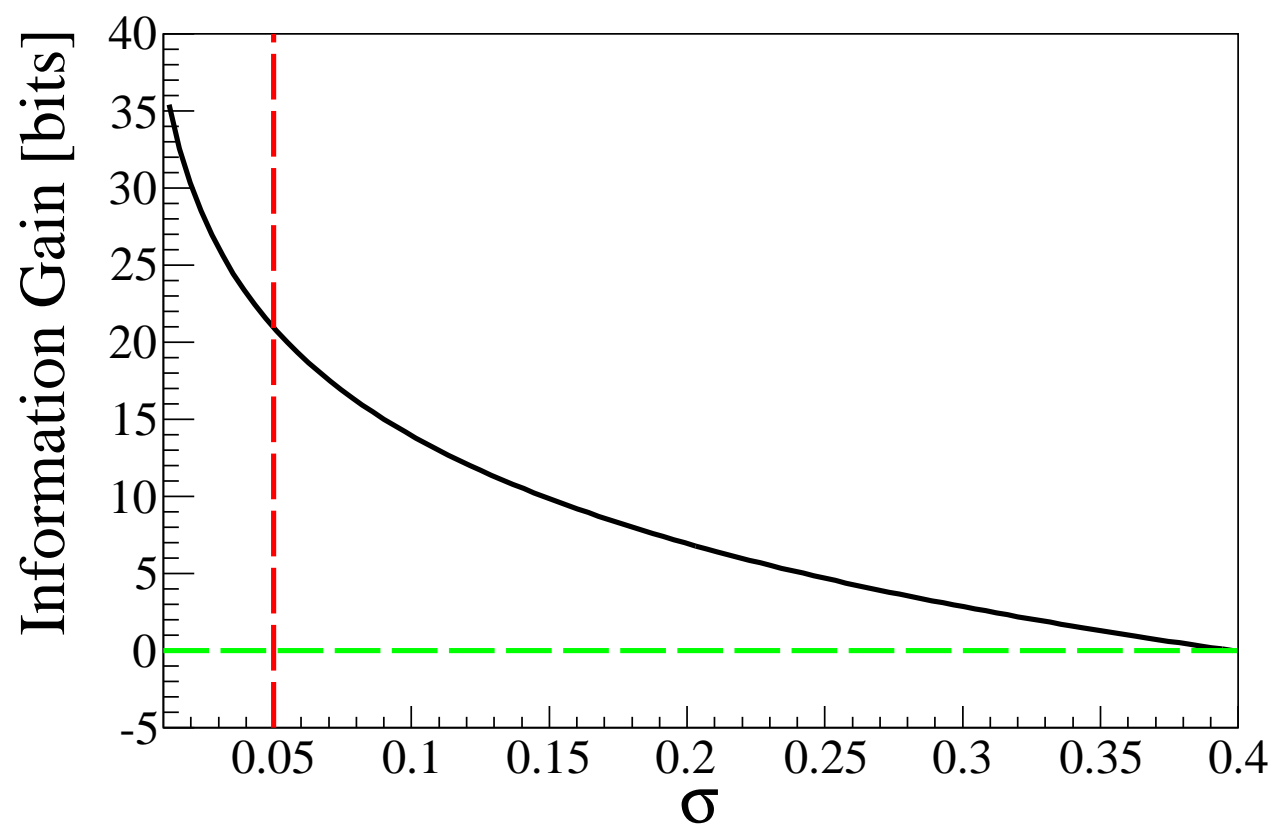

Figure 1: (Color online) Rough guide to information gain as a function of the standard deviation $\sigma$ in the real and imaginary parts of the amplitudes.

number of dimensions in this system indicates the use of Monte Carlo techniques, and a simple implementation of this is as follows.

Sample points are generated randomly in amplitude space with uniform density on the surface of the unit 7-sphere. The number of points $N_{0}$ needs to be sufficiently large to minimize Monte Carlo sampling uncertainty. For each point, the observables are evaluated according to the algebra of table $\amalg$ in the appendix. The use of random values of amplitudes was described in [4] in order to establish, for combinations of observables, the limits of regions in observable space that are allowed by postivity constraints, and using this a a guide for deriving inequalities. The present work goes further by not only taking into account these positivity constraints, but also estimating the PDFs of the combinations of observables. One can then simulate the process of measuring an observable by weighting all the points by another PDF representing the measured observable.

In practice, the PDF of an asymmetry is likely to be something like a beta distribution (or a Gaussian approximation thereof). For illustrative purposes, however, we can use a simple top-hat function, which for a single observable is equivalent to reducing the range of values from $[-1,1]$ to $[r-\delta, r+\delta]$, where $r$ is the measured result with some 
uncertainty $\pm \delta$. If the uniform probability density on a multi-dimensional surface $S$ is $p\left(x_{i}\right) d\left\{x_{i}\right\}=d\left\{x_{i}\right\} / S$. The entropy of a uniform distribution in a volume $S$ is then

$$
H=-\int \frac{1}{S} \log \left(\frac{1}{S}\right) d\left\{x_{i}\right\}=\log S,
$$

as illustrated by the value for the 7-sphere in Eq. (4).

If the surface is reduced by a cut, say from $S_{0}$ to $S_{1}$, the probability density will be uniform in $S_{1}$ and zero otherwise, so the gain in information is simply the log of the ratio of the two surface areas:

$$
I=\log S_{0}-\log S_{1}
$$

When cuts representing the measurement of a combination of observables are imposed, the number of remaining points $N_{1}$ is an estimate of the remaining volume, so

$$
I=\log N_{0}-\log N_{1} .
$$

So in order to gain the 21 bits of information, the surface area in amplitude space (and hence the number of points) needs to be reduced by a factor of $2^{21} \approx 10^{6}$.

This is best illustrated with a simple example, such as the measurement of one polarization observable, recoil polarization, say. Figure 2 shows in the light shade the distribution of $10^{6}$ points when sampling is done uniformly in amplitude space. The dark shaded region shows 126045 points selected when a simulated measurement of $R=-0.4 \pm 0.1$ is selected. The result is an information gain of $6 \log _{2} 10-\log _{2} 126045=2.988 \pm 0.003$ bits, where the uncertainty is an estimate of the Monte Carlo error. So we can expect that a measurement of one polarization observable to an accuracy of $\pm 10 \%$ will give us about 3 bits of information.

Note that the "uncut" or prior distribution is quadratic in shape, not only for recoil polarization, but for all observables. This is a consequence of the observables being bilinear combinations of the amplitudes.

\section{SIMULATING COMBINATIONS OF MEASUREMENTS}

\section{A. Measuring all $\mathcal{S}$-type observables}

For the extraction of amplitudes, it is usually assumed that the $\mathcal{S}$-type observables $(B, R$ and $T)$ have to be measured. Let us examine how much information one gains by 


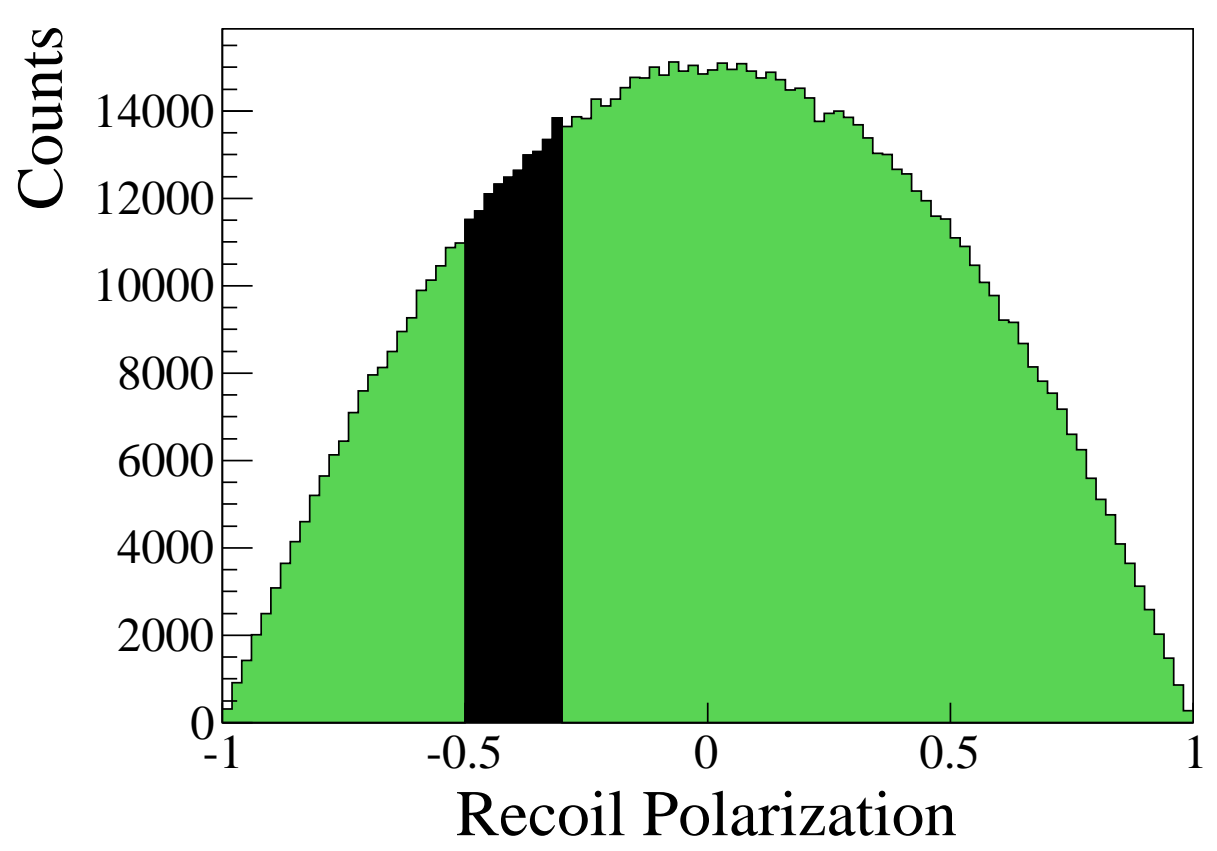

Figure 2: (Color online) Distribution of values of recoil polarization from the uniform PDF in amplitude space. Shaded region represent the possible values remaining after a "measurement".

making such measurements.

As shown in [4, 5], the constraints among observables

$$
|T-R| \leq 1-B ; \quad|T+R| \leq 1+B
$$

carve out a tetrahedron inside a cube $[-1,+1]^{3}$ in BRT-space. To approximate a measurement of $B, R$ and $T$, we define a spherical region, of radius $r$, i.e.

$$
(B-x)^{2}+(R-y)^{2}+(T-z)^{2} \leq r^{2}
$$

where $(x, y, z)$ are the coordinates of the sphere centre. This spherical cut can be moved to various points within the tetrahedron, and the effect on the distributions of magnitudes and phases studied.

A typical example is depicted in Fig. 3. The bottom left panel shows a projection of the BRT distributions, which highlights the tetrahedral region. Recall that the points in the light shaded region have been initially sampled over amplitude space, so this represents a projection into $B R T$-space, and affirms the constraints defined by Eq. (8). The points in the dark sphere are those selected by the choice of cut region. The radius of 


\begin{tabular}{l|l} 
Position in $B R T$ tetrahedron & Magnitude information \\
\hline Center & All magnitudes equal \\
Mid-point of face & One magnitude small, others large and equal \\
Mid-point of edge & Two magnitudes small and equal, other two large and equal \\
Vertex & One magnitude large, others small and equal
\end{tabular}

Table I: Guide to relative size of magnitudes for various positions within the BRT tetrahedron

the spherical cut is 0.1 , which is equivalent in information gain to a measured accuracy in each observable of better that \pm 0.05 (see later). It is unlikely, when statistical and systematic uncertainties are taken into account, that experiments will be able to determine observables to much greater accuracy than this.

In the example of Fig. 3, the spherical cut is just touching the midpoint of one of the tetrahedron faces. The top row shows the magnitudes of the amplitudes, and it is clear that values for each one can now be estimated. Note, however, that there is much greater uncertainty in $\left|a_{2}\right|$ than in the other ones.

The relative phase angles are displayed in the remaining panels. While only three relative angles are independent, all six possibilities are shown. This is because, for situations in which the magnitudes of two amplitudes $a_{i}$ and $a_{j}$ are almost equal (as in this case), very small uncertainties in the relative phase of the two amplitudes with respect to a third $\left(\theta_{i k}\right.$ and $\left.\theta_{j k}\right)$ could lead to very large uncertainties in their relative phase $\theta_{i j}$. It is to be expected that there should be no relative phase information for transversity amplitudes if only $\mathcal{S}$-type measurements are made, and this is apparent from the distributions in Fig. 3. The observed increase towards $\theta_{i j}=0^{\circ}$ is due to the fact that the relative angles are formed from the difference of two uniform random variables.

By examining the variations in the distributions of magnitudes and phases for different positions in the $B R T$ tetrahedron, one can deduce some general heuristics governing the relation between what we shall call a $B R T$ measurement and the magnitudes $\left|a_{i}\right|$. These are listed in table I.

Returning to the information gain from a $B R T$ measurement, if we assume that the sampled points in amplitude space project into a uniformly dense $B R T$ tetrahedron, the 

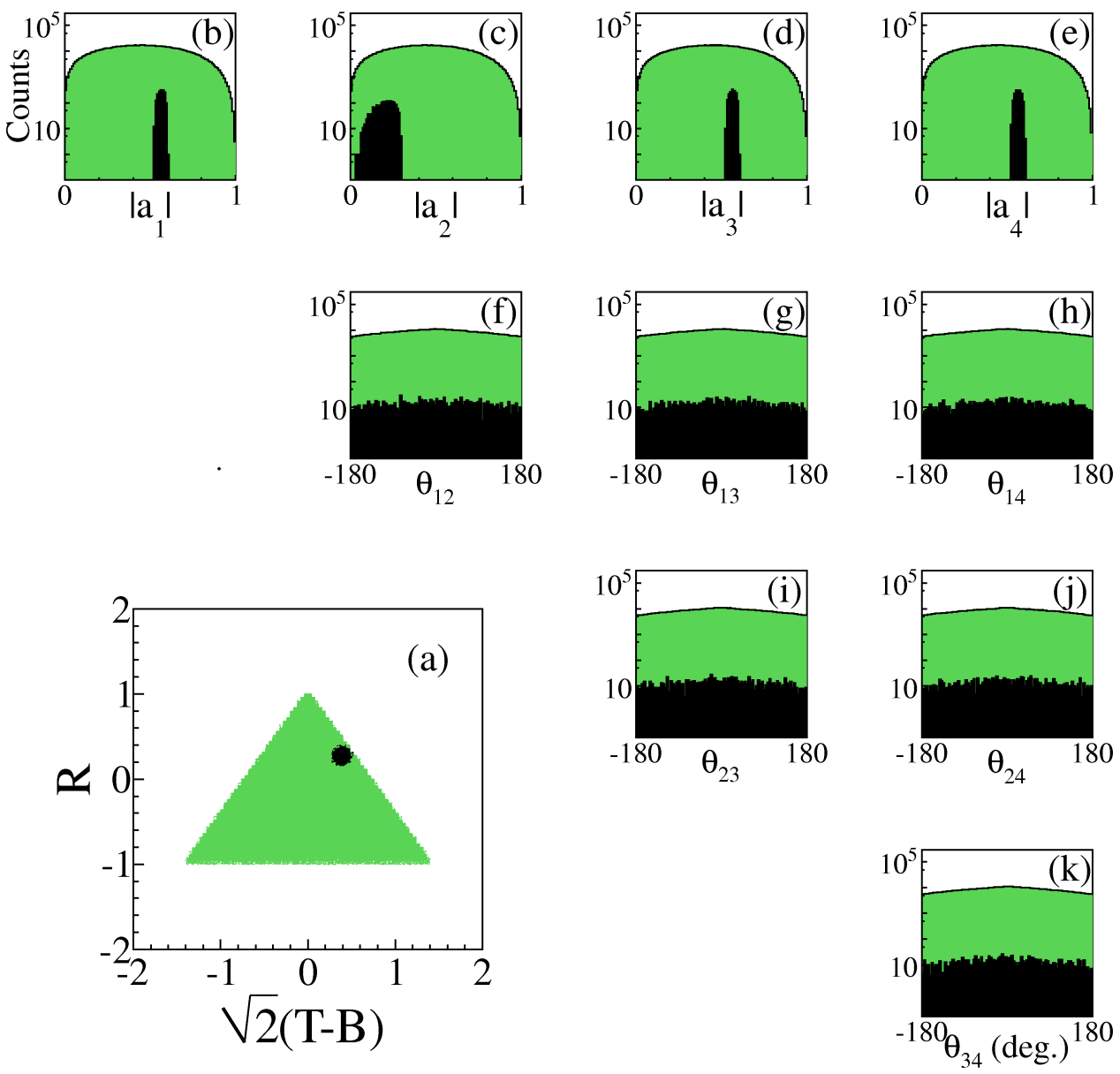

Figure 3: (Color online) Light shade - uniform sample of amplitude space; dark shade - region surviving cut. Panel (a) is the projection of BRT tetrahedron, (b)-(e) show the magnitudes of the amplitudes and the other panels are the distributions of relative phase angles (in degrees).

entropy before a measurement is

$$
H_{\text {tetra }}=\log 8-\log 3,
$$

i.e. the volume is a third of the cube $[-1,+1]^{3}$. A 3D gaussian, with symmetric widths $\sigma$ has entropy

$$
H_{3 D \text { Gaussian }}=\frac{3}{2} \log (2 \pi e)+3 \log \sigma,
$$

from Eq. (5). To establish an equivalent spherical cut, one can use the entropy of a sphere of radius $r$ (inside tetrahedron),

$$
H_{\text {sphere }}=\log \left(\frac{4}{3} \pi r^{3}\right)
$$


and equate Eq. (9) and Eq. (10) to establish a relationship between $r$ and $\sigma$ :

$$
\log r-\log \sigma=\frac{1}{2} \log (2 \pi e)-\frac{1}{3} \log \left(\frac{4}{3} \pi\right),
$$

from which we have $r \approx 2.564 \sigma$, so a spherical cut of radius 0.1 is equivalent to Gaussian errors on $B, R$ and $T$ with $\sigma=0.039$. Using these figures, the predicted information gain is 9.31 bits for any position of the spherical cut within the tetrahedron. For the case depicted in Fig. 3, the estimated information gain is $9.28 \pm 0.02$.

It can be readily demonstrated that the estimates of information gain are equal to the predicted value of 9.31 (to within sampling errors) for all the classes of position listed in table I, hence verifying the assumption that the $B R T$ tetrahedron is uniformly sampled. So for real experiments, knowing the uncertainties of the measured values of $B, R$ and $T$ allows a calculation of information gain, irrespective of the size of the measured values.

\section{B. Towards Extraction of Amplitudes}

Compared to the original guideline of 21 bits, we can see that the measurement of just $\mathcal{S}$-type observables leaves a lot of information to be gained. With just under 10 bits, the magnitudes can be determined to roughly $10 \%$ accuracy, but to determine relative phases to better than, say, a 16th $\left(=2^{-4}\right)$ of the full angular range will require an additional 4 bits for each one. Adding this information together brings us to 22 bits, one greater than the original estimate. One might imagine that measurement of an additional four double polarization observables would now be sufficient, given that individual measurements can gain about 3 bits (see section 【). However, the complicated relations among observables now conspire against this.

Chiang and Tabakin [2] systematically listed the possible combinations of observables that would lead to a "complete" set; there are a large number of them. They took one example set which showed a counter-example to the claim in [1] that completeness could only be attained if five observables are measured, of which four should not be from the same $\mathcal{B R}$-, $\mathcal{B} \mathcal{T}$ - or $\mathcal{R} \mathcal{T}$-set. In that example, $F, G$ and $L_{x}$ were taken to be measured, and whereas Ref. [1] claimed that $E$ and $L_{z}$ were needed, Ref. [2] asserted that only $T_{x}$ was necessary.

Using the scheme already outlined, we may examine what happens when simulated 
measurements are made of the same sets of observables. The BRT measurements are all assumed to have been made, but to study whether the results for information gain depend on the measure values, four possible cases of position in the measured BRT tetrahedron have been used: center, mid-face, mid-edge and vertex. They give a representative sample of all possible cases, and due to the tetrahedral symmetry only one mid-face, mid-edge and vertex needs to be considered. For each of the four cases, $10^{5}$ events were generated within the defined spherical sub-region of the BRT tetrahedron. These were selected by rejection from an intial uniform sample over amplitude space (the 7-sphere). In order to simulate possible measurements of $F, G$ and $L_{x}$, a \pm 0.1 cut on the generated points around a central value of each observable was imposed. The central values are shown for each case in table II. Relatively large values were chosen for clarity of illustration, and note that the same values of $F, G$ and $L_{x}$ could not be used for each case because of the interdependency of these observables with the chosen BRT values.

For each set of $B R T$ values, three cases were studied for combinations of further measurements: $T_{x}$ only (choice of Ref. [2]), $E$ and $L_{z}$ (choice of Ref. [1]) and $T_{x}, E$ and $L_{z}$. Again, a \pm 0.1 cut on the generated points around a central value of each observable is applied. The results for information gain are shown in the penultimate column of table II.

Several points are apparent from the results displayed. It is clear that the more measurements that are made, the more information that is gained. It is also clear that the information gain is dependent on the assumed measured $B R T$ values. Recall that the information gain obtained when measuring only $B R T$ values was independent of position in the BRT tetrahedron; the difference is again due to the interdependency among observables. When the information gain is greater that 13 , the number of points surviving the cuts is 10 or less, so the estimates are of limited accuracy.

All the cases of combinations of observables that are listed in table $\amalg$ have previously been proved to result in mathematically complete sets. With the introduction of simulated experimental uncertainty, however, this can no longer be taken to be adequate. The last column of the table (headed "Ambiguity?") indicates whether there are identifiable, unambiguous values of both magnitudes and relative phases of the amplitudes. The midface case, where $T_{x}$ only has been measured in addition to the common set of observables, is illustrated in Fig. 4. Despite the few surviving points, it is fairly clear that there are no 


\begin{tabular}{|c|c|c|c|c|c|c|c|c|}
\hline BRT position & $F$ & $G$ & $L_{x}$ & $E$ & $L_{z}$ & $T_{x}$ & Information (bits) & Ambiguity? \\
\hline \multirow{3}{*}{ Center } & 0.4 & 0.4 & 0.3 & - & - & 0.7 & $11.4 \pm 0.2$ & $\mathrm{Y}$ \\
& 0.4 & 0.4 & 0.3 & 0.3 & 0.3 & - & $12.7 \pm 0.3$ & $\mathrm{~N}$ \\
& 0.4 & 0.4 & 0.3 & 0.3 & 0.3 & 0.7 & $13.2 \pm 0.3$ & $\mathrm{~N}$ \\
\hline \multirow{3}{*}{ Mid-Face } & 0.4 & -0.4 & 0.4 & - & - & 0.4 & $11.1 \pm 0.1$ & $\mathrm{Y}$ \\
& 0.4 & -0.4 & 0.4 & 0.7 & -0.7 & 0.4 & $12.7 \pm 0.3$ & $\mathrm{~N}$ \\
\hline \multirow{5}{*}{ Mid-Edge } & 0.4 & 0.4 & 0.4 & 0.2 & -0.7 & - & $13.6 \pm 0.4$ & $\mathrm{~N}$ \\
& 0.4 & 0.4 & 0.4 & 0.2 & -0.7 & -0.7 & $13.6 \pm 0.4$ & $\mathrm{~N}$ \\
\hline \multirow{2}{*}{ Vertex } & 0.4 & 0.4 & 0.4 & - & - & 0.3 & $8.8 \pm 0.1$ & $\mathrm{Y}$ \\
& 0.4 & 0.4 & 0.4 & 0.3 & 0.2 & - & $11.1 \pm 0.1$ & $\mathrm{~N}^{*}$ \\
& 0.4 & 0.4 & 0.4 & 0.3 & 0.2 & 0.3 & $11.5 \pm 0.2$ & $\mathrm{~N}^{*}$ \\
\hline
\end{tabular}

Table II: Results of simulated measurements for different combinations of observables. The values of each observable are all defined with a \pm 0.1 cut.

three relative phase angles that have a single cluster of points, and so an unambiguous extraction of amplitudes would not be possible.

For the cases listed in table $\amalg$ with $\mathrm{N}^{*}$ for ambiguity, this indicates that while there is just one identifiable cluster of points in the distributions of relative phases, the spread in possible points is greater than $10 \%$ of the full angular range; i.e. there may be no quadrant ambiguity, but there remains a considerable uncertainty.

It appears, from this very small sample of possible outcomes, that for measurement of double polarization observables an information gain of about 12 bits is required. Combining this number with that from the measurement of $B R T$ ( $\sim 10$ bits), this leads us to a crude, but very helpful conclusion: only when the total information gain from polarization observables is greater than about 21 bits should it be possible to extract amplitudes from experimental measurements. This condition will apply irrespective of which particular set of observables have been measured, since information gain is simply a measure how of much one has compressed the original uniform PDF in amplitude space. This number is also in line with the crude calculation given in Eq. (7), where the real and 

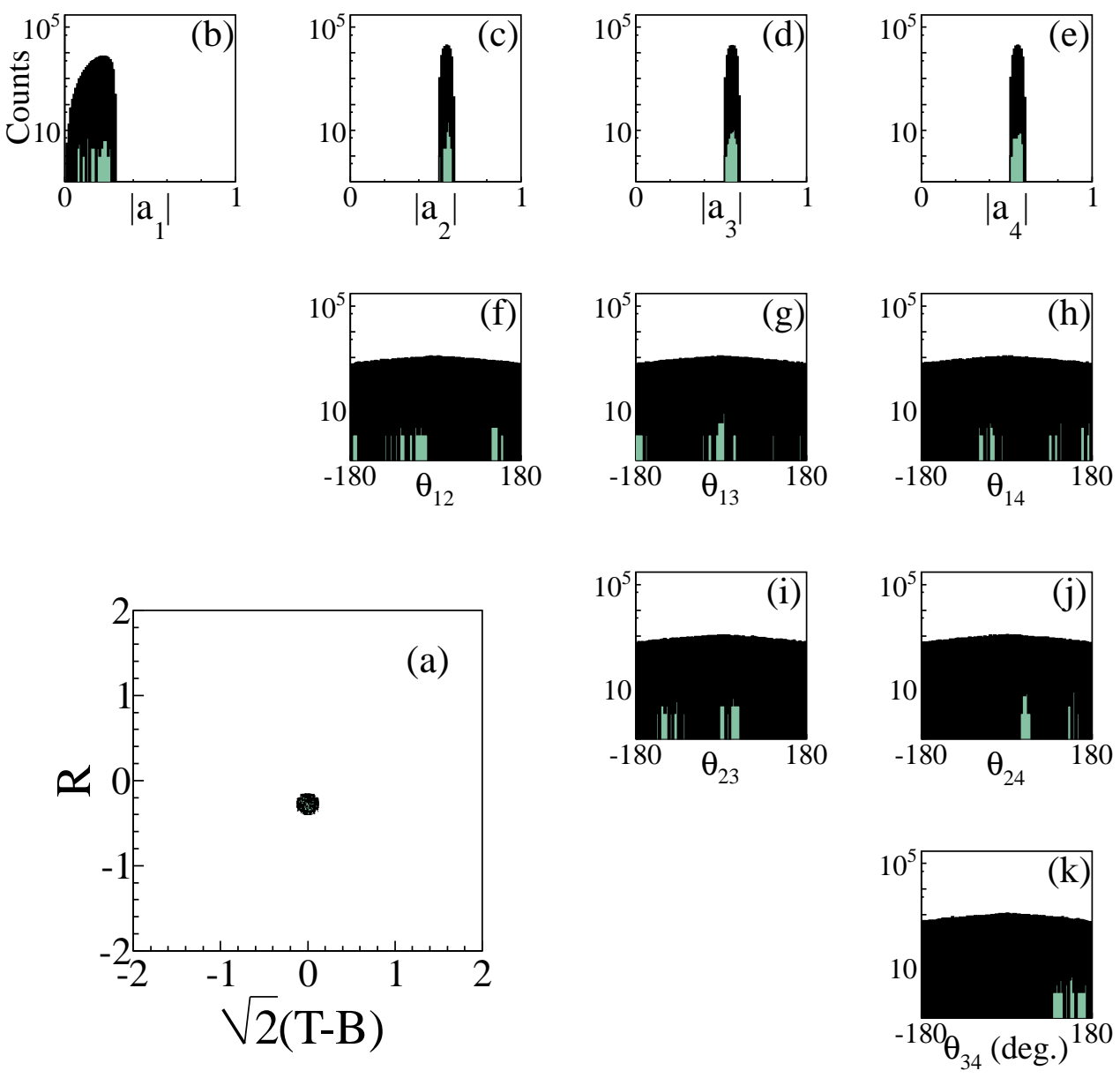

Figure 4: (Color online) Example of residual ambiguity in relative phases after measurement of set $F, G, L_{x}$ and $T_{x}$. Light shade - uniform sample of amplitude space; Dark shade - region within BRT tetrahedron; Light shade - points surviving cuts in $F, G, L_{x}$ and $T_{x}$. Labels (a)-(k) are as in Fig. 3 .

imaginary parts of the amplitudes were assumed to be extracted to an accuracy of 0.05.

The scheme outlined above uses "cuts" in the space of possible observables to simulate the act of measurement, and the reduced observable space is projected back into amplitude space to calculate the associated entropy. This is a crude, but effective, means of relating the observable-space PDF to the amplitude-space PDF. To apply the idea of information gain to the results of actual experiments, this scheme will have to be modified. When the measurement of a set of observables is made, the result will be an approximately multi-dimensional Gaussian PDF over the range of those observables. A PDF in amplitude space can be constructed by sampling uniformly over all amplitude space, 
calculating the value of the observables for each sample point then weighting them with the values of the experimentally determined observable PDF. The resulting amplitude PDF can be made arbitrarily accurate, depending on the number of sample points, but for calculating information gains of 21 bits, $\mathcal{O}\left(10^{7}\right)$ points may be needed.

One final comment related to practical experiments is in order. It is clear that for extraction of amplitudes, it is essential to be able to polarize photon beams and targets, and to detect recoil polarization. Given that all three components of the reaction require this technological effort, the most obvious strategy is to worry less about which combination of observables to measure, and more about trying to measure as many as possible, with as great an accuracy as possible. The theoretical work in Refs. [1, 2] is, however, still a useful guide to selecting the combinations of observables that will most efficiently lead to an information gain of 21 bits. The information measure (3) can be used in the design of experiments to provide an estimate of the degree of accuracy (and hence the integrated luminosity) required for amplitude extraction.

\section{COMPARISON OF MODELS WITH DATA}

Having established how to estimate the quantity of information contained in measured data, can the measured data be used to extract information about the parameters of an individual model?

An individual model will depend on some input parameters, $\xi$, say (e.g. coupling constants). Quite often, the comparison of model calculations to data is used to extract "best fit" values for the input parameters, $\xi^{\star}$. We can use the information gain for measured data to tell whether a fit to the new data will yield an improved knowledge of input parameters, compared to prior information. Prior to a fit procedure, knowledge about the possible values $\xi$ will be encoded in a PDF $p(\xi, M)$, where $M$ is there as a reminder that this quantity depends on a model. The amplitude PDF of the model, given a specified set of input parameters is $p\left(x_{i} \mid \xi, M\right)$, where as before $x_{i}$ represents the real and imaginary parts of the amplitudes. The total prior PDF of the model is an integral over the range of input parameters

$$
p\left(x_{i} \mid M\right)=\int p\left(x_{i} \mid \xi, M\right) p(\xi, M) d \xi,
$$

so a model entropy $H_{\text {model }}$ can be evaluated by plugging the model prior PDF into Eq. 
(3). Then only if $H_{\text {measured }} \ll H_{\text {model }}$ is there likely to be a significant improvement in the knowledge of the input parameters when the model is fitted to the data.

\section{v. CONCLUSIONS}

In this article a measure of information content, based on Shannon entropy, was introduced and has been applied to measurement of polarization observables in pseudo-scalar meson photoproduction. Using the uncertainties in the measurements, the information entropy of the four amplitudes can be calculated. It is assumed that a suitably accurate determination of the cross-section has been made, which gives an overall scale factor to the amplitudes.

An important finding is that, when allowing for a realistic but small measurement uncertainty, measuring only a mathematically complete set of observables is not enough to guarantee the extraction of amplitudes. Instead, a rule of thumb, based on quantifying information gain of about 21 bits for each point in $\left\{W, \theta_{C M}\right\}$, is likely to be a more robust guide.

An extension of the work presented here is likely to be applicable to other reactions in which information content could determine whether measurements will be adequate to extract physically meaningful results. Examples such as the extraction of generalized parton distributions from DVCS-like asymmetries, or inferring the details of the nucleonnucleon interaction from the database of scattering observables may be fruitful areas of investigation.

\section{Acknowledgments}

This work was supported by the United Kingdom's Science and Technology Facilities Council.

[1] I. S. Barker, A. Donnachie, and J. K. Storrow, Nucl. Phys. B 95, 347 (1975).

[2] W.-T. Chiang and F. Tabakin, Phys. Rev. C55, 2054 (1997).

[3] C. E. Shannon, The Bell System Technical Journal 27, 379 (1948). 
[4] X. Artru, M. Elchikh, J.-M. Richard, J. Soffer, and O. V. Teryaev, Physics Reports 470, 1 (2009).

[5] X. Artru, J.-M. Richard, and J. Soffer, Phys. Rev. C75, 024002 (2007).

[6] This departure from the usual conventions is to avoid confusion between $\Sigma$-particle and $\Sigma$ beam asymmetry, as well as $P$-photon polarization and $P$-recoil.

\section{Appendix A: Definitions of Observables}

In this work, transversity amplitudes are used, although none of the results depend on using this basis. The transversity basis refers to the projection of the recoil or target spin along the normal to the scattering plane. The four amplitudes for pseudo-scalar meson photoproduction are

$$
\begin{array}{ll}
b_{1}=\langle+|\mathcal{M}|+\perp\rangle ; & b_{2}=\langle-|\mathcal{M}|-\perp\rangle ; \\
b_{3}=\langle+|\mathcal{M}|-\|\rangle ; & b_{4}=\langle-|\mathcal{M}|+\|\rangle .
\end{array}
$$

Here, The labels $\|$ and $\perp$ stand for the photon $E$-vector (linear polarisation) parallel to and normal to the scattering plane respectively. In order to deal only with asymmetries these amplitudes are divided by the cross-section to give

$$
a_{i}=\frac{b_{i}}{\sqrt{\left|b_{1}\right|^{2}+\left|b_{2}\right|^{2}+\left|b_{3}\right|^{2}+\left|b_{4}\right|^{2}}}
$$

The experimentally measured observables are then related to the $a_{i}$ as shown in table III, Whereas the double poalrization observables are denoted in a conventional way, $R, B$ and $T$ have been chosen to represent Recoil, Beam and Target asymmetries. 


\begin{tabular}{ccc} 
Observable & Type & Amplitude Combination \\
\hline \hline$R$ & Single & $\left|a_{1}\right|^{2}-\left|a_{2}\right|^{2}+\left|a_{3}\right|^{2}-\left|a_{4}\right|^{2}$ \\
$B$ & $\left|a_{1}\right|^{2}+\left|a_{2}\right|^{2}-\left|a_{3}\right|^{2}-\left|a_{4}\right|^{2}$ \\
$T$ & $\left|a_{1}\right|^{2}-\left|a_{2}\right|^{2}-\left|a_{3}\right|^{2}+\left|a_{4}\right|^{2}$ \\
\hline$E$ & Beam-Target & $2 \Re\left(a_{1} a_{3}^{\star}+a_{2} a_{4}^{\star}\right)$ \\
$F$ & & $2 \Im\left(a_{1} a_{3}^{\star}-a_{2} a_{4}^{\star}\right)$ \\
$G$ & $2 \Im\left(a_{1} a_{3}^{\star}+a_{2} a_{4}^{\star}\right)$ \\
$H$ & & $-2 \Re\left(a_{1} a_{3}^{\star}-a_{2} a_{4}^{\star}\right)$ \\
\hline$C_{x}$ & Beam-Recoil & $-2 \Im\left(a_{1} a_{4}^{\star}-a_{2} a_{3}^{\star}\right)$ \\
$C_{z}$ & & $2 \Re\left(a_{1} a_{4}^{\star}+a_{2} a_{3}^{\star}\right)$ \\
$O_{x}$ & & $2 \Re\left(a_{1} a_{4}^{\star}-a_{2} a_{3}^{\star}\right)$ \\
$O_{z}$ & & $2 \Im\left(a_{1} a_{4}^{\star}+a_{2} a_{3}^{\star}\right)$ \\
\hline$T_{x}$ & Target-Recoil & $2 \Re\left(a_{1} a_{2}^{\star}-a_{3} a_{4}^{\star}\right)$ \\
$T_{z}$ & & $2 \Im\left(a_{1} a_{2}^{\star}-a_{3} a_{4}^{\star}\right)$ \\
$L_{x}$ & & $-2 \Im\left(a_{1} a_{2}^{\star}+a_{3} a_{4}^{\star}\right)$ \\
$L_{z}$ & & $2 \Re\left(a_{1} a_{2}^{\star}+a_{3} a_{4}^{\star}\right)$ \\
\hline & &
\end{tabular}

Table III: Definition of observable quantities in terms of the (scaled) amplitudes. 